\title{
CARACTERIZAÇÃO DO RUÍDO AMBIENTAL URBANO EM MARINGÁ, PARANÁ
}

\author{
LIMA, Bruna Tamara de \\ Universidade Estadual de Maringá (UEM), e-mail: bruna_tamara@hotmail.com \\ SOARES, Paulo Fernando \\ Universidade Estadual de Maringá (UEM), e-mail: pfsoares@vem.br
}

\begin{abstract}
RESUMO
O ruído é uma das principais fontes de desconforto para o homem. A coexistência de atividades econômicas e residências é um dos problemas a serem avaliados, pois, a economia cresce e traz desenvolvimento, mas é capaz de promover o desconforto acústico à população. O trabalho, apresentado em disciplina de mestrado, visou caracterizar o ruído em uma porção central de Maringá, por meio de medições in loco para os cálculos dos níveis sonoros. Ao confirmar a extrapolação dos decibéis estabelecidos em norma e legislação, evidencia-se a necessidade de se planejar melhor o desenvolvimento das cidades e da proposição de alternativas que possam, de maneira viável, mitigar o problema.
\end{abstract}

Palavras-chave: Planejamento urbano, Polvição sonora, Conforto ambiental.

\begin{abstract}
Noise is one of the main sources of discomfort for man. The coexistence of economic activities and residences is one of the problems to be evaluated, because, the economy grows and brings development, but it is able to promote acoustic discomfort to the population. The work, presented in a master's degree, aimed to characterize the noise in a central portion of Maringá, by means of in loco measurements for the calculation of the sound levels. In confirming the extrapolation of the decibels established in standard and legislation, it is evident the need to better plan the development of cities and the proposition of alternatives that can, in a feasible way, mitigate the problem.
\end{abstract}

Keywords: Urban planning, Noise pollution, Environmental comfort.

\section{INTRODUÇÃO}

O ruído tem figurado como fator de grande de preocupação para a saúde e qualidade de vida das populações urbanas, pois o desenvolvimento econômico das cidades leva à necessidade da realização de atividades que expõe os cidadãos a um ruído de fundo constante - principalmente o tráfego veicular (MENDONÇA et al., 2013; GERAGHTY; O'MAHONY, 2016; GAJARDO et al., 2016). Segundo Amorim et al. (2017), o ruído ambiental corresponde a superposição de ruídos, que, geralmente, têm naturezas e origens diferentes, dos quais nenhum é objeto de estudo isoladamente, mas aquele originário no tráfego veicular destaca-se.

De acordo com Mendonça et al. (2013), o ruído ambiental urbano pode comprometer a integridade do meio ambiente e a saúde das populações. Por

LIMA, B. T.; SOARES, P. F. Caracterização do ruído ambiental urbano em Maringá, Paraná. In: SIMPÓSIO BRASILEIRO DE QUALIDADE DO PROJETO NO AMBIENTE CONSTRUÍDO, 6., 2019, Uberlândia. Anais... Uberlândia: PPGAU/FAUeD/UFU, 2019. p. 1166-1171. DOI https://doi.org/10.14393/sbqp19106 
esse motivo, é importante que o ruído ambiental urbano seja estudado e mitigado.

A caracterização do ruído ambiental urbano pode ser formulada a partir de medições deste. Assim, a seleção de áreas problemáticas é uma maneira mais viável, tanto em termos espaciais quanto temporais, de caracteriza-lo (MURPHY; KING, 2014).

Assim, esta pesquisa tem como objetivo caracterizar o ruído percebido em uma área localizada no centro de Maringá. O local selecionado é uma área de interesse para estudos a respeito de ruído ambiental dada a concorrência de atividades comerciais diversas, tráfego veicular intenso e de edifícios residenciais.

\section{MATERIAIS E MÉTODOS}

O Município de Maringá está situado no noroeste do Estado do Paraná e é o polo de uma Região Macroeconômica de 25 municípios. A população da cidade era de 357077 habitantes em 2010 e estima-se que seja de 417010 habitantes em 2018 (IBGE, 2018; PREFEITURA DE MARINGÁ, 2018).

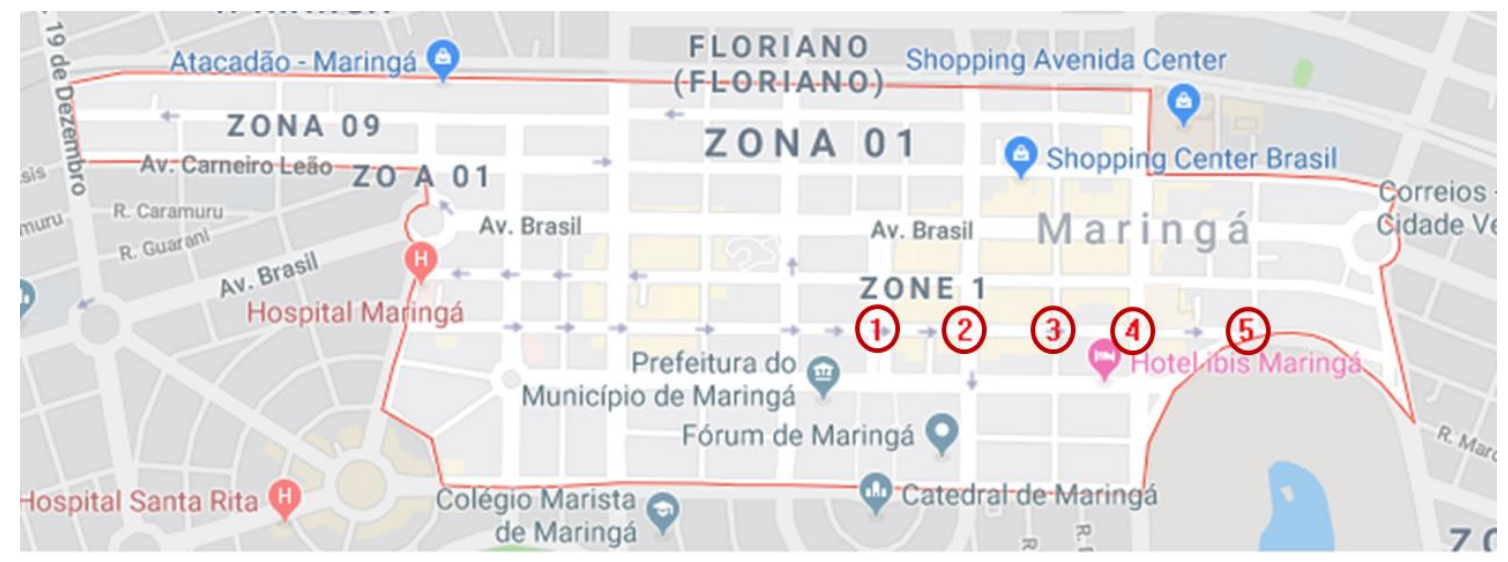

Figura 1 - Zona 01 de Maringá e pontos de medição -

Fonte: GOOGLE (2018), modificado pelos autores

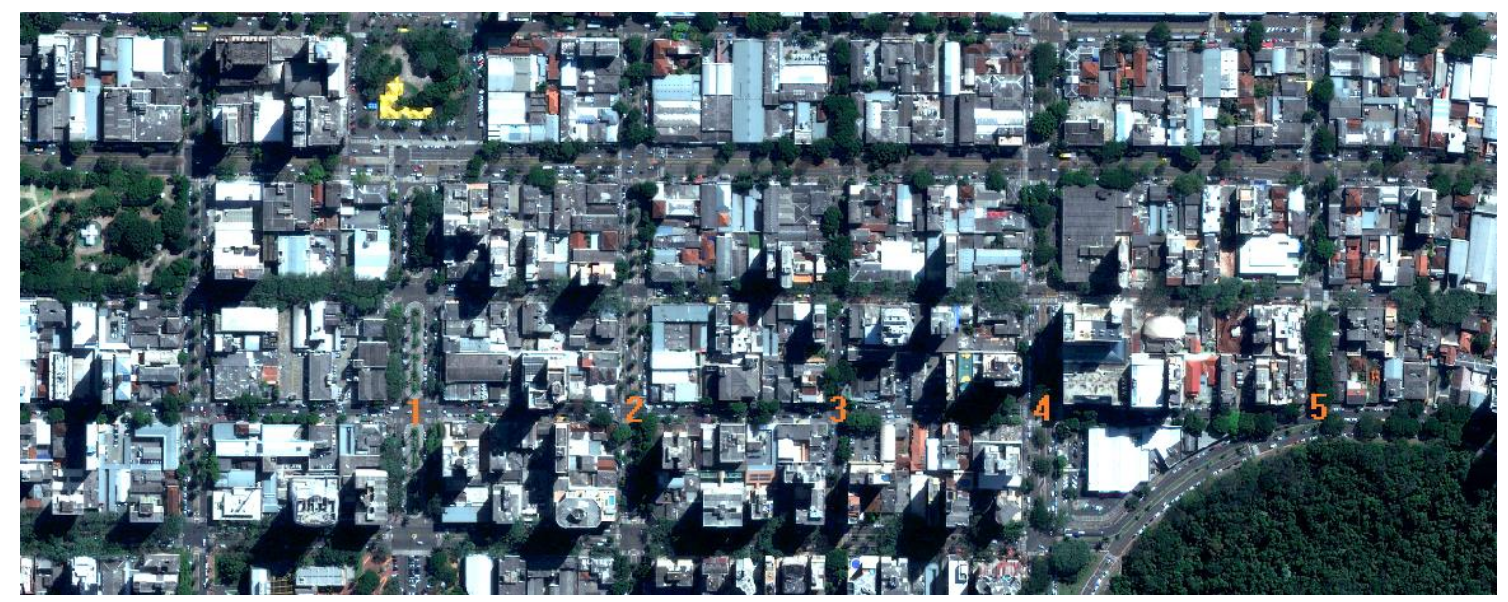

Figura 2 - Pontos de medição -

Fonte: PREFEITURA DE MARINGÁ (2019) 
Devido a relevância econômica do município, escolheu-se a região central para avaliação do ruído ambiental. O local selecionado pertence a Zona 01 maringaense, onde são permitidas atividades de comércio e serviços, residências, pequenas indústrias e atacados, que não sejam nocivas ao homem (MARINGÁ, 2013).

A Zona 01 de Maringá possui 10946 habitantes e sua área tem quase todo seu solo utilizado e ocupado por instalações comerciais e de serviços. A maior parte das residências está abrigada nos pisos superiores de edifícios cujos pavimentos térreos abrigam atividades econômicas (IBGE, 2018; PREFEITURA DE MARINGÁ, 2018).

Pode-se, então, enquadrar a zona em questão como uma "Área mista, com vocação comercial e administrativa"; portanto, poderia ser exposta a, no máximo, $60 \mathrm{~dB}(\mathrm{~A})$ no período diurno (das 7:00 as 20:00 horas), conforme a ABNT NBR 10151 (ABNT, 2000) e a legislação municipal (MARINGÁ, 1998).

Para a realização do estudo, foram executadas medições de ruído nos cruzamentos da Rua Néo Alves Martins com as avenidas Getúlio Vargas e Herval, com a Rua Piratininga, com a Avenida São Paulo e com a Rua Octávio Perioto, (intersecção 1, 2, 3, 4 e 5, respectivamente), como apresentado nas Figuras 1 e 2.

A via cujas intersecções foram monitoradas foi selecionada por contemplar instalações de serviços e comércios diversas (como lojas variadas, escritórios, imobiliárias, restaurantes e até um shopping center), dos quais muitos são edifícios residenciais nos pavimentos superiores.

As leituras foram tomadas em novembro de 2018, em dias de terças, quartas e quintas-feiras (por serem dias laborais), às 08:00, às 12:00 e às 18:00 horas (pois são os horários de pico).

Para as aferições foi utilizado o medidor de nível de pressão sonora DL 4200. Conforme a NBR 10151 (ABNT, 2000), o microfone do aparelho estava protegido por espuma, posicionado a 2,00 metros de qualquer tipo de barreira e a 1,50 metros do solo (fixado sobre um tripé).

Dos dados obtidos pelas leituras calculou-se o nível de pressão sonora equivalente (LAeq) por meio da equação 1 (ABNT, 2000):

$$
L_{\text {Aeq }}=10 \log \frac{1}{n} \sum_{i=1}^{n}\left(10^{\frac{L i}{10}}\right)
$$

Em que, Li é o nível de pressão sonora, em $\mathrm{dB}(\mathrm{A})$ de cada medição do ruído e n corresponde ao número total de leituras.

Foram calculados também os níveis estatísticos L90, L50 e L10, que correspondem aos níveis sonoros excedidos em $90 \%, 50 \%$ e $10 \%$ do tempo de medição, respectivamente. Estes descritores são determinados ao serem ordenados de modo crescente os valores aferidos e efetuando-se as frequências acumuladas (BISTAFA, 2006).

O cálculo do índice Ltráfego foi realizado segundo a equação 2 (LO BOSCO; LEONARDI; SCOPELLITTI, 2006): 


$$
L_{\text {Atráfego }}=52+10 \log \frac{Q}{q}
$$

Em que $Q$ é o número de veículos e q é a distância da via.

Calculou-se também o índice de ruído de tráfego (TNI - Traffic Noise Index), conforme a equação 3 (LANGDON; SCHOLES, 1968; BRESSANE et al., 2016).

$$
L_{A T N I}=4 x\left(L_{10}-L_{90}\right)+L_{90}-30
$$

\section{RESULTADOS E DISCUSSÕES}

Os valores médios calculados de LAeq, L10, L50, L90, LAtráfego e LAtni são apresentados no Quadro 1.

\begin{tabular}{|c|c|c|c|c|c|c|}
\hline \multicolumn{7}{|c|}{ 08h00min } \\
\hline Intersecção & LAeq & L10 & L50 & L90 & LAtráfego & LAtni \\
\hline 1 & 68,0 & 70,0 & 62,0 & 57,0 & 72,0 & 78,0 \\
\hline 2 & 70,0 & 72,0 & 68,0 & 61,0 & 78,0 & 76,0 \\
\hline 3 & 67,0 & 70,0 & 63,0 & 57,0 & 71,0 & 82,0 \\
\hline 4 & 70,0 & 73,0 & 68,0 & 61,0 & 76,0 & 76,0 \\
\hline 5 & 68,0 & 70,0 & 66,0 & 62,0 & 73,0 & 66,0 \\
\hline \multicolumn{7}{|c|}{$12 \mathrm{~h} 00 \mathrm{~min}$} \\
\hline Intersecção & LAeq & L10 & L50 & L90 & LAtráfego & LAtni \\
\hline 1 & 68,0 & 68,18 & 62,8 & 58,1 & 72,43 & 68,42 \\
\hline 2 & 73 & 72,6 & 66,3 & 61,4 & 75,09 & 76,2 \\
\hline 3 & 69 & 68,54 & 62 & 56,76 & 72,17 & 73,88 \\
\hline 4 & 74 & 73,01 & 67,8 & 62 & 76,63 & 76,04 \\
\hline 5 & 69 & 69,1 & 64,35 & 59,9 & 72,63 & 66,7 \\
\hline \multicolumn{7}{|c|}{$18 \mathrm{~h} 00 \mathrm{~min}$} \\
\hline Intersecção & LAeq & L10 & L50 & L90 & LAtráfego & LAtni \\
\hline 1 & 66,0 & 69,0 & 61,0 & 57,0 & 72,0 & 72,0 \\
\hline 2 & 69,0 & 71,0 & 66,0 & 60,0 & 77,0 & 77,0 \\
\hline 3 & 65,0 & 68,0 & 61,0 & 54,0 & 71,0 & 79,0 \\
\hline 4 & 70,0 & 73,0 & 68,0 & 61,0 & 76,0 & 77,0 \\
\hline 5 & 65,0 & 68,0 & 62,0 & 55,0 & 70,0 & 79,0 \\
\hline
\end{tabular}

Quadro 1 - Níveis de pressão sonora e níveis estatísticos (em dB(A))

Verifica-se que os níveis de ruído ultrapassam o limite estabelecido em norma e legislação. Salvo em dois pontos, o nível de pressão sonora equivalente (LAeq) assim como os descritores estatísticos $L 10$ e $L 50$ e os índices relacionados ao tráfego veicular (LAtráfego e LAtni), apresentam valores que extrapolam os $65 \mathrm{~dB}(\mathrm{~A})$ estabelecido em norma (ABNT 10151, 2000) e determinado pela legislação do Município de Maringá (MARINGÁ, 1998). Essa superação do limite de tolerância é verificada em quase todos os pontos, dias e horários. Ou seja, pode-se dizer que a área é crítica no que diz respeito ao ruído ambiental. 
O nível estatístico de $90 \%$, o 290 , é o único que apresenta valores abaixo de 65 $\mathrm{dB}(\mathrm{A})$, com níveis de ruído em torno dos $60 \mathrm{~dB}(\mathrm{~A})$. Isso significa que é possível atender a norma e legislação, pois este descritor caracteriza o ruído verificado em $90 \%$ do tempo de medição.

Por serem pontos localizados na região central da cidade, é plausível atribuir ao trânsito a maior parcela de contribuição na geração de tal ruído. Ao que se soma não terem sido constatadas outras fontes de ruído significativas (grandes obras ou atividades industriais, por exemplo) nos períodos de medição. Dessa forma, fica evidente a necessidade de ações de planejamento e intervenção, principalmente em relação ao fluxo de veículos na região e/ou alterações na dinâmica urbana, que levem a uma minimização do problema.

\section{CONCLUSÃO}

A caracterização do ruído ambiental é um interessante instrumento de planejamento urbano, como o estudo de caso demonstrou, pois os níveis de ruído obtidos ultrapassam o limite estabelecido em norma e legislação em uma área em que considerável número de pessoas é impactado. Pode-se considerar que a pesquisa vai ao encontro dos 17 Objetivos de Desenvolvimento Sustentável da Agenda 2030 para o Desenvolvimento Sustentável, propostos pela ONU em 2015 (AGENDA 2030, 2018), porque a problemática afeta a saúde das populações expostas e faz parte de um planejamento urbano para a segurança das pessoas e sustentabilidade urbana. Mostrando a contribuição do trabalho e indicando a necessidade de estudos futuros que apresentem alternativas para minimizar o problema.

\section{AGRADECIMENTOS}

Trabalho realizado com apoio da Coordenação de Aperfeiçoamento de Pessoal de Nível Superior - Brasil (CAPES) - Código de Financiamento 001.

\section{REFERÊNCIAS}

AMORIM, A. E. B.; DURANTE, L. C.; VILELA, J. C.; CALLEJAS, I. J. A. Previsão do ruído ambiental urbano devido à implantação do Modal Veículo Leve sobre Trilhos (VLT) por meio de simulação computacional. INTERAÇÕES, Campo Grande, v. 18, n. 4, p. 81-97, out./dez. 2017.

ASSOCIAÇÃO BRASILEIRA DE NORMAS TÉCNICAS (ABNT). NBR 10151: Acústica Avaliação do ruído em áreas habitadas, visando o conforto da comunidade Procedimento. Rio de Janeiro, jun. 2000.

NBR 10152: Acústica - Níveis de pressão sonora em ambientes internos a edificações. Rio de Janeiro, nov. 2017.

AGENDA 2030. O que é a Agenda 2030? Disponível em: <http://www.agenda2030.c om.br/>. Acesso em: 13 nov. 2018.

BISTAFA, S. R. Acústica Aplicada ao Controle do Ruído. 1.ed. São Paulo: Blucher, 2006. 
BRESSANE, A.; MOCHIZUKI, P. S.; CARAM, R. M.; ROVEDA, J. A. F. Sistema de apoio à avaliação de impactos da polvição sonora sobre a saúde pública. Cadernos de Saúde Pública, v. 32, n. 5, mai. 2016.

GAJARDO, C. P.; MORILLAS, J. M. B.; GOZALO, G. R.; VÍLCHEZ-GÓMEZ, R. Can weekly noise levels of urban road traffic, as predominant noise source, estimate annual ones?. The Journal of the Acoustical Society of America, v. 140, n. 5 , nov. 2016.

GERAGHTY, D.; O'MAHONY, M. Urban Noise Analysis Using Multinomial Logistic Regression. Journal of Transportation Engineering, v. 142, n. 6, jun. 2016.

GOOGLE. Google Maps. Disponível em: <https://www.google.com/maps/plac e/Zona+01,+Maring\%C3\%A 1+-+PR/@-23.4219542,-51.9583824,14z/data=!3m1 !4b 1!4m5!3m4! 1 s0x94ecd72bc9c30971:0xa27ecc2ee758d4e5!8m2!3d-23.4194131!4 d-51.9394994>. Acesso em: 30 out. 2018.

INSTITUTO BRASILEIRO DE GEOGRAFIA E ESTATÍSTICA (IBGE). Maringá. Disponível em: <https://cidades. ibge.gov.br/brasil/pr/maringa/panorama>. Acesso em: 21 out. 2018.

LANGDON, I. D.; GRIFFITHS, F. J. Subjective Response to Road Traffic Noise! Journal of Sound and Vibration, v. 8, n. 1, p. 16-32, jul. 1968.

LO BOSCO, D.; LEONARDI, G.; SCOPELLITTI, F. Metodologie per lo studio dell'inquinamento acustico da traffico veicolare. Università Degli Studi Mediterranea di Reggio Calabria, Facoltà di Ingegneria, Dipartimento di Informatica, Matematica, Elettronica e Trasporti. Calábria, Itália, 2006.

MARINGÁ. Lei Complementar n.॰ 951, de 26 de julho de 2013. Autoriza o Poder Executivo a Regulamentar os Usos Permissíveis com Fundamento no Artigo $6^{\circ}$, $\S$ $1^{\circ}$, Da Lei Complementar $n^{\circ} 888$, de 26 de Julho De 2011.

MENDONÇA, A. B. D.; SURIANO, M. T.; SOUZA, L. C. L.; VIVIANI, E. Classes de quadras urbanas determinadas pelos níveis de ruídos. urbe. Revista Brasileira de Gestão Urbana, v. 5, n. 2, Curitiba, jul./dez., 2013.

MURPHY, E.; KING, E. Environmental Noise Pollution: Noise Mapping, Public Health, and Policy. 1 ed. Oxford: Elsevier, 2014.

PREFEITURA DE MARINGÁ. Maringá. Disponível em: <http://www2.maringa.pr.go v.br/turismo/?.cod=nossa-cidade/3>. Acesso em: 21 out. 2018.

PREFEITURA DE MARINGÁ. Maringá Turística. Disponível em: <http://geoproc.ma ringa.pr.gov.br:8090/PORTALCIDADAO/>. Acesso em: 23 out. 2018. 